\title{
Placental Transmogrification of the Lung
}

\author{
Jin Woo Kim, $M D^{1}$, Il Hwan Park, $M D^{2}$, Woocheol Kwon, $M D^{1}$, Min Seob Eom, MD ${ }^{3}$, Young Ju Kim, $M D^{1}$, \\ Joong Hwan Oh, MD²
}

Departments of ${ }^{1}$ Radiology, ${ }^{2}$ Thoracic and Cardiovascular Surgery, and ${ }^{3}$ Pathology, Yonsei University Wonju College of Medicine, Wonju 220-701, Korea

Placental transmogrification is a very rare lung disease, where the alveoli resemble the chorionic villi of placenta, and this change is a characteristic finding. A 31-year-old female patient presented with cough and dyspnea that had begun 2 weeks prior to admission. Along with giant bulla found in the left upper lung field, subsegmental consolidation was also identified in the lingular segment on plain chest radiograph and CT scan. Wedge resection was performed to remove the bulla. Pathologic examination of the resected bulla revealed destruction of the normal structures and characteristic villous and papillary changes. These changes led to a diagnosis of placental transmogrification. We made an encounter of an unusual placental transmogrification which had different image findings from other reported transmogrification cases. Thus, we report an atypical placental transmogrification case where both consolidation and giant bulla coexist.

Index terms: Lung; Placenta; Chorionic villi; Emphysema

\section{INTRODUCTION}

Placental transmogrification, first described in 1979, is a rare and benign disease of the lung, whose cause is still unknown $(1,2)$. Radiologically, placental transmogrification of the lung mainly shows bullous changes and presents, very rarely, as cysts or nodules $(2,3)$. Pathology of the disease characteristically shows papillary structures similar to placental villi surrounding the pulmonary epithelium

Received November 30, 2012; accepted after revision August 4, 2013.

Corresponding author: Il Hwan Park, MD, Department of Thoracic and Cardiovascular Surgery, Yonsei University Wonju College of Medicine, 20 Ilsan-ro, Wonju 220-701, Korea.

- Tel: (8233) 741-1322 • Fax: (8233) 7422-0666

- E-mail: nicecs@yonsei.ac.kr

This is an 0pen Access article distributed under the terms of the Creative Commons Attribution Non-Commercial License (http://creativecommons.org/licenses/by-nc/3.0) which permits unrestricted non-commercial use, distribution, and reproduction in any medium, provided the original work is properly cited.
(3). Through this study, we a thoroughly review previous literatures on placental transmogrification, a rare disease entity, and report an unusual case of the disease with both a bullous change and consolidation.

\section{CASE REPORT}

A 31-year-old female patient presented with cough and mild dyspnea to the outpatient department. She had a history of being diagnosed with pulmonary tuberculosis 7 years ago, which she had completely recovered after 6 months of antituberculosis drug regimen. There was no other notable history. Dullness and decreased breathing sound at the left side was noted after auscultation. Complete blood count revealed no abnormal finding, and no acid-fast bacilli was discovered on the sputum culture test. Plain chest radiography showed giant bulla in the left lung and subsegmental consolidation in the left lower lung field. Chest computed tomography (CT) scan revealed the left upper lobe of the lung being replaced by bullae 

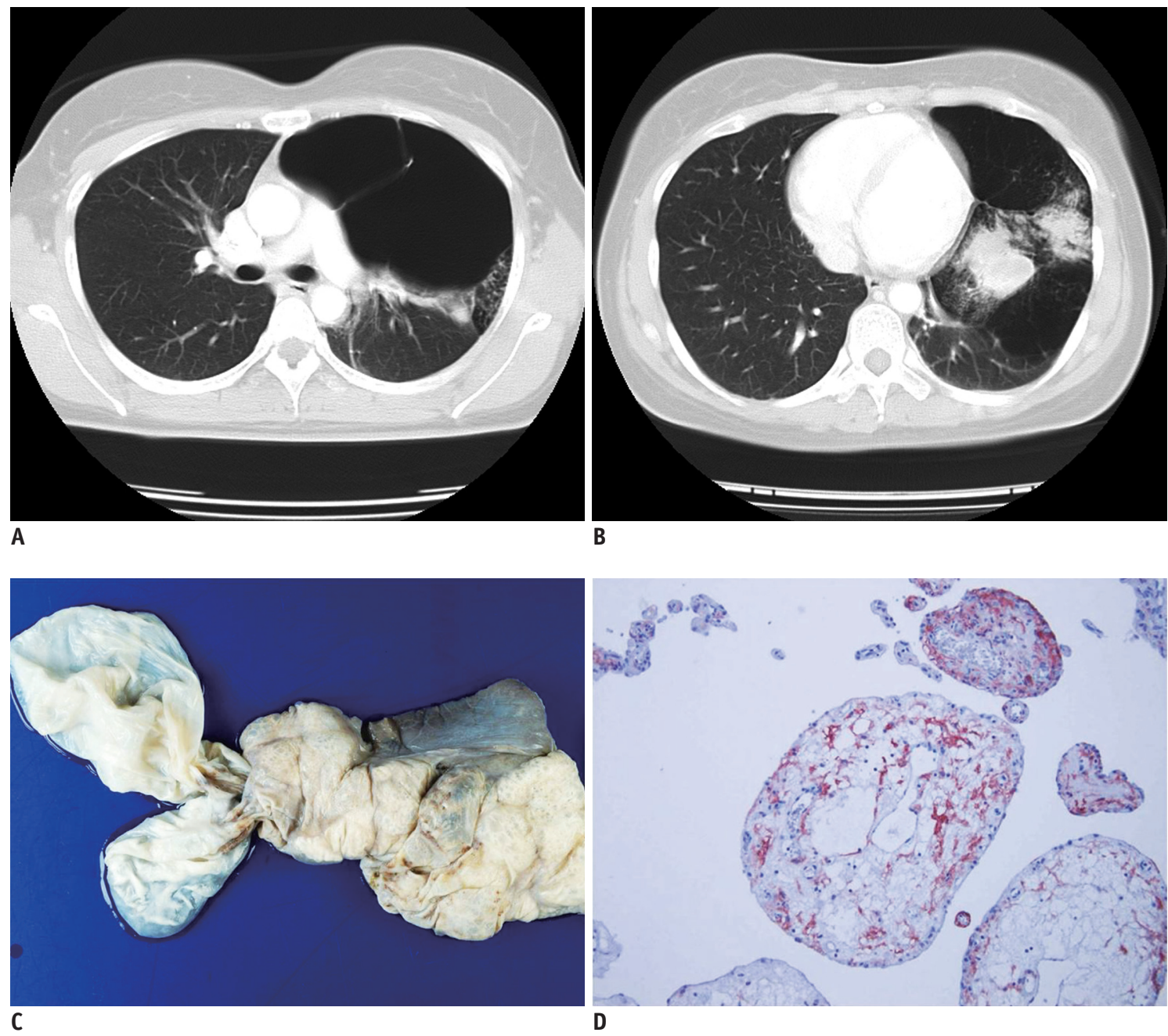

Fig. 1. Thirty-one-year-old female patient with large bullae and subsegmental consolidation in left upper lobe.

A. Preoperative chest computed tomography shows multiple giant bullae in left upper lobe with resultant mediastinal shifting. B. Subsegmental consolidation in left lingular segment has broad contact with bullae. C. Pleural surface shows several massively dilated bullae, largest one measuring $11 \times 5 \times 3 \mathrm{~cm}$ in size. D. On high power, villous structures are composed of simple cuboidal cell linings and edematous cores. Immunohistochemical stain for CD-10 is positive only in interstitial cells (IHC, $\times 400)$.

of various sizes, and subsegmental consolidation had broad contacted with bullae at the lingular segment of the left upper lobe (Fig. 1A, B). The right lung showed no abnormality. The patient's pulmonary function test signified moderate obstructive respiratory failure. Wedge resection of the left lung using video-assisted thoracoscopy was performed under the preoperative diagnoses of giant bullae and pulmonary emphysema. During operation, the left lower lobe was found to be severely compressed by the bullae. Gross examination of the wedge resected lung was $15 \times 9 \times$ $4 \mathrm{~cm}$ in size with dilated giant bullae (Fig. 1C). Spongiform emphysema was observed at the cut surface, and there were partial solid changes and fibrotic progression. Examination of the solidified sites by light microscope indicated general emphysematous changes with destruction of the alveolar wall in addition to numerous tissues replaced by structures similar to the chorionic villi. The villous structure was adjacent to, or in some cases adhered to, the destroyed alveolar wall and interlobular septa. Immunohistochemical staining of the epithelium lining the villous structure was partly positive for thyroid transcription factor- 1 , and the interstitial cells at the core was positive for CD-10, D240 and vimentin (Fig. 1D). There was no human chorionic gonadotropin positive cell.

The chest tube was removed 8 days after surgery. Pathologic examination of the surgical specimen reported placental transmogrification of the lung. Chest CT scan was taken to rule out the possibility of remnant bullae or transmogrified tissue. The giant bullae at the left upper lobe were removed, but some fibrotic tissue still remained. 
Additional left upper lobectomy was planned to remove the remaining tissue in order to minimize recurrence. However, the patient did not complain of any symptoms and refused further operation. She was explained about the possibility of recurrence and scheduled visits to the outpatient clinic on a regular basis for follow-ups.

\section{DISCUSSION}

Placental transmogrification of the lung, or pulmonary placental transmogrification, is also referred to as placentoid bullous lesion. It is known to be a very rare disease $(1,2)$ with less than 30 adequately documented cases. Clinically, placental transmogrification usually occurs in men between 20 to 50 years old; however, our case was even a more rare case due to the occurrence in a female patient. It may be asymptomatic or perhaps associated with pneumothorax or chronic obstructive lung disease (4).

The etiology and pathogenesis still remain unclear. It may be congenital, but no placental transmogrification has been reported in children (5). Placental transmogrification is not only associated with cystic or emphysematous lung lesion, but also with pulmonary fibrochondromatous hamartomas and pulmonary lipomatosis. Thus, the radiologic presentation can be bullous change or pulmonary lung nodules $(6,7)$.

The papillary placentoid and villous structures resembling chorionic villi, which are characteristically seen in this patient, are known as placental transmogrification. A villous transformation is thought to arise from edema and fibrosis of pulmonary strands of severe emphysema or giant bullous emphysema. Lipomatosis is also implicated as a cause due to the expression of fat tissues inside the villi as well as frank lipomatosis in the placental villi and in the interstitium of the lung (4). Others report the proliferation of interstitial clear cell as primary occurrence and emphysema-like cystic changes as secondary occurrence (8). Placental transmogrification of the lung is distinguished from typical emphysema by the tendency to occur in the younger age group along with the local progression of the disease (8).

Previous articles had classified the radiologic findings into 3 patterns. The most common manifestation is bullous emphysema pattern $(1-3,8)$. Next, the disease is expressed on radiography with a mixed pattern of thin-walled cystic lesion and nodule $(6,7,9)$. Rarely, the radiologic finding shows a solitary nodule pattern (3). The disease itself is a rare disease entity, and our case had even more unusual findings of both giant bulla and subsegmental consolidation patterns.

Radiologically, differential diagnosis of the lesion includes cystic or bullous lung disease, such as bullous emphysema, particularly giant bullous emphysema (vanishing lung syndrome), and solitary pulmonary lung nodules, such as hamartoma $(3,9)$.

Bullous emphysema shows similar imaging characteristics to placental transmogrification. Placental transmogrification usually has a unilateral giant bullous change. However, bullous emphysema generally demonstrates a diffuse bilateral lung involvement $(8,9)$. In addition, although bullous emphysema can have rapid progression of lung parenchyma destruction in drug abuse, acquired immune deficiency syndrome or autoimmune disease, it commonly affects old age with a history of smoking and alpha-1 antitrypsin deficiency and develops over a long period of time (5). In particular, giant bullous emphysema is also referred to as primary bullous emphysema or vanishing lung syndrome, which is characterized by bulla occupying at least one third of the hemithorax. It may most closely resemble the image characteristics similar to placental transmogrification.

Other radiologic findings of placental transmogrification are emphysema with pulmonary nodules or solitary pulmonary nodule patterns. In rare cases, these solid pulmonary nodules contain air and fat (3). The radiologic differential diagnosis of the nodule includes pulmonary hamartoma. However, the image finding alone has limited value to differentiate the lesion from placental transmogrification. Therefore, making the diagnosis requires the confirmation of pathology.

In summary, the image finding of placental transmogrification presents unilateral large bullous emphysema with or without an associated nodule or consolidation. From a clinical standpoint, making an accurate preoperative diagnosis is difficult. Although it is an extremely rare disease, it must be ruled out in patients with unilateral bullous lesion who do not have high risk of bullous emphysema.

\section{REFERENCES}

1. McChesney T. Placental transmogrification of the lung: a unique case with remarkable histopathologic features. Lab Invest 1979;40:245-246 
2. Horsley WS, Gal AA, Mansour KA. Unilateral giant bullous emphysema with placental transmogrification of the lung. Ann Thorac Surg 1997;64:226-228

3. Ferretti GR, Kocier M, Moro-Sibilot D, Brichon PY, Lantuejoul S. Placental transmogrification of the lung: CT-pathologic correlation of a rare pulmonary nodule. AJR Am J Roentgenol 2004;183:99-101

4. Fidler ME, Koomen M, Sebek B, Greco MA, Rizk CC, Askin FB. Placental transmogrification of the lung, a histologic variant of giant bullous emphysema. Clinicopathological study of three further cases. Am J Surg Pathol 1995;19:563-570

5. Theile A, Wex P, Müller KM. [Placentoid malformation of the lung as differential diagnosis of localized emphysema]. Pneumologie 1997;51:550-554

6. Park ES, Han J, Koh WJ, Lee KS, Kim J, Seo J, et al. Placental transmogrification of the lung: a brief case report. Korean $\mathrm{J}$ Pathol 2008;42:308-310

7. Xu R, Murray M, Jagirdar J, Delgado Y, Melamed J. Placental transmogrification of the lung is a histologic pattern frequently associated with pulmonary fibrochondromatous hamartoma. Arch Pathol Lab Med 2002;126:562-566

8. Cavazza A, Lantuejoul S, Sartori G, Bigiani N, Maiorana A, Pasquinelli $G$, et al. Placental transmogrification of the lung: clinicopathologic, immunohistochemical and molecular study of two cases, with particular emphasis on the interstitial clear cells. Hum Pathol 2004;35:517-521

9. Shapiro M, Vidal C, Lipskar AM, Gil J, Litle VR. Placental transmogrification of the lung presenting as emphysema and a lung mass. Ann Thorac Surg 2009;87:615-616 